\title{
Global Inhibition and Stimulus Competition in the Owl Optic Tectum
}

\author{
Shreesh P. Mysore, ${ }^{\star}$ Ali Asadollahi, ${ }^{\star}$ and Eric I. Knudsen \\ Department of Neurobiology, Stanford University, Stanford, California 94305
}

Stimulus selection for gaze and spatial attention involves competition among stimuli across sensory modalities and across all of space. We demonstrate that such cross-modal, global competition takes place in the intermediate and deep layers of the optic tectum, a structure known to be involved in gaze control and attention. A variety of either visual or auditory stimuli located anywhere outside of a neuron's receptive field (RF) were shown to suppress or completely eliminate responses to a visual stimulus located inside the RF in nitrous oxide sedated owls. The essential mechanism underlying this stimulus competition is global, divisive inhibition. Unlike the effect of the classical inhibitory surround, which decreases with distance from the RF center and shapes neuronal responses to individual stimuli, global inhibition acts across the entirety of space and modulates responses primarily in the context of multiple stimuli. Whereas the source of this global inhibition is as yet unknown, our data indicate that different networks mediate the classical surround and global inhibition. We hypothesize that this global, cross-modal inhibition, which acts automatically in a bottom-up manner even in sedated animals, is critical to the creation of a map of stimulus salience in the optic tectum.

\section{Introduction}

In all classes of vertebrate species, from fish to mammals, the optic tectum (OT) is crucially involved in the processes that direct an animal's gaze toward a stimulus (Wurtz and Goldberg, 1971; Robinson, 1972; Schiller and Stryker, 1972; Roucoux et al., 1980; Hikosaka and Wurtz, 1985a; Viévard et al., 1986; Knudsen et al., 1993; Masino and Knudsen, 1993). The OT, usually referred to as the superior colliculus in mammals, has also been implicated in stimulus selection for controlling the locus of attention. Inactivation of the intermediate layers of the superior colliculus ( $\mathrm{SCi}$ ) in monkeys results in deficits in stimulus selection based on perceptual judgments (McPeek and Keller, 2004). In a wide variety of nonprimate species, inactivation of the intermediate and deep layers of the OT (OTi-d) causes a profound neglect of stimuli located in the inactivated portion of the OT space map (Sprague and Meikle, 1965; Kirvel et al., 1974; Raczkowski et al., 1976; Dean and Redgrave, 1984; Overton and Dean, 1988; Knudsen et al., 1993). Conversely, focal electrical microstimulation applied to the SCi improves the ability of monkeys to detect and discriminate visual stimuli specifically at the location represented by the microstimulation site (Carello and Krauzlis, 2004; Cavanaugh and Wurtz, 2004; Müller et al., 2005).

Received July 31, 2009; revised Dec. 2, 2009; accepted Dec. 18, 2009.

This research was supported by the Dean's Postdoctoral Fellowship, Stanford University (S.P.M.) and National Institutes of Health Grant 9R01 EY019179-29 (E.I.K.). We are grateful to Steve Baccus for critically reading this manuscript. We thank Alex Goddard and Devarajan Sridharan for discussions and for critically reading this manuscript. S.P.M. thanks Dan Winkowski for discussions and training. We also thank Joe Bergan, Kristin Maczko, and Ilana Witten for their help with stimulus presentation protocols.

*S.P.M. and A.A. contributed equally to this work.

Correspondence should be addressed to Shreesh P. Mysore, 299 W Campus Drive, Department of Neurobiology, Stanford University, Stanford, CA 94305. E-mail: shreesh@stanford.edu.

DOI:10.1523/JNEUROSCI.3740-09.2010

Copyright $\odot 2010$ the authors $\quad 0270-6474 / 10 / 301727-12 \$ 15.00 / 0$
Despite the evidence that the OTi-d contributes to stimulus selection for gaze and attention, principles of stimulus selection in the OTi-d have not been established. Psychophysical and neurophysiological experiments have demonstrated that stimulus selection is a competitive process (Desimone and Duncan, 1995; Baddeley, 2003; Knudsen, 2007). When the competition is not biased by top-down influences, it is based on the relative physical distinctiveness (salience) of sensory stimuli in the world. The competition acts across sensory modalities and across the entirety of space to select the next most salient stimulus. Models of spatial attention posit that this competition occurs within specialized modules, called salience maps, consisting of mutually competing elements that are tuned for different locations in space and that respond over wide ranges of stimulus feature values (Koch and Ullman, 1985; Treisman, 1988; Cave and Wolfe, 1990; Olshausen et al., 1993; Findlay and Walker, 1999; Itti and Koch, 2001; Wolfe, 2003).

There is substantial evidence that the OTi-d may contain a salience map. Strong competitive interactions have been observed in the OTi-d between the representations of widely separated stimuli in goldfish (Schellart et al., 1979), pigeons (Frost et al., 1981), and cats (Rizzolatti et al., 1973, 1974; Meredith and Stein, 1986). Moreover, this competition acts across sensory modalities: neuronal responses to either a visual, auditory, or somatosensory stimulus located inside of a neuron's receptive field (RF) can be suppressed by either a visual, auditory, or somatosensory stimulus located outside of the RF.

Although these properties are consistent with those expected to underlie stimulus selection for gaze control or attention, many aspects of the competition that is expressed in the OTi-d have not been explored parametrically. Here, we report the effects of activating OTi-d neurons in the barn owl with pairs of discrete stimuli. We describe the spatial extent, profile, and nature of the inhibitory effects of a second stimulus (either visual or auditory) 
on responses to a visual stimulus located inside the visual RF. We contrast the properties of global inhibition, revealed using pairs of stimuli, with those of the classical inhibitory surrounds, revealed using single stimuli. The competitive interactions we observe in the OTi-d are consistent with those predicted for a salience map by models of spatial attention (Itti and Koch, 2000).

\section{Materials and Methods}

Animals, surgical preparation, and neurophysiology

Animals. Seven adult barn owls were used in this study. Materials and methods for bird care and for surgery were approved by the Stanford University Institutional Animal Care and Use Committee and were in accordance with the National Institutes of Health and the Society for Neuroscience guidelines for the care and use of laboratory animals.

Surgical preparation. Owls were prepared for multiple electrophysiological experiments. Before the first experiment, an owl was anesthetized with isoflurane (1\%) and a mixture of nitrous oxide and oxygen (45:55), and a head bolt was mounted at the rear of the skull. Plastic cylinders that permitted access to the brain were implanted over the optic tectum bilaterally. Polysporin antibiotic ointment was applied to the exposed brain surface, and the recording chambers were sealed. All wounds were cleaned with Betadine and infused with a local anesthetic. After recovering from surgery, the owl was returned to its flight room.

On the day of an experiment, the owl was anesthetized with isoflurane $(1 \%)$ and a mixture of nitrous oxide and oxygen (45:55) and was placed in a restraining tube in a prone position within a sound-attenuating booth. The head was secured to a stereotaxic device, and the visual axes were aligned relative to a calibrated tangent screen (the eyes of the owl are essentially stationary in the head). Owls were maintained on just the mixture of nitrous oxide and oxygen from the time when they were secured in the booth through the end of the experiment.

Neurophysiology. Epoxy-coated tungsten microelectrodes (4-6 M $\Omega$ at $1 \mathrm{kHz}$ ) were used to record extracellularly from single and multiple units (sites) in the intermediate and deep layers (layers 11-15) of the OT. These layers were identified by distinctive neuronal responses that have been associated reliably with these layers based on lesion-reconstruction experiments (DeBello and Knudsen, 2004). Visual and auditory stimuli were presented for $250 \mathrm{~ms}$. Spikes were recorded from 400 to $1000 \mathrm{~ms}$ before stimulus onset until 500-1250 ms after stimulus onset. Interstimulus intervals (ISIs) ranged from 2.5 to $5.0 \mathrm{~s}$, and the number of trials repeated at a stimulus location ranged from 10 to 25 . Spike times were stored using Tucker-Davis hardware (RA-16) controlled by customized MATLAB (The MathWorks) software.

\section{Visual stimulation}

Visual stimuli were created using customized MATLAB software (courtesy of J. Bergan, Harvard University, Cambridge, MA) and presented (Mitsubishi $\mathrm{XD} 300 \mathrm{U}$ projector) on a calibrated tangent screen at a distance of 35 $\mathrm{cm}$ from the eyes. The owl was positioned so that the visual axes were in the horizontal plane aligned with $0^{\circ}$ elevation and $0^{\circ}$ azimuth of the screen. All locations are given in double pole coordinates of azimuth, relative to the midsagittal plane, and elevation, relative to the visual plane.

Spatial tuning curves. Spatial tuning to visual stimuli was measured by presenting a $1^{\circ}$ radius, negative contrast (black on a gray background) dot, moving at $20 \%$ s. Tuning to visual azimuth (or elevation) was first estimated by presenting 10-15 series of moving dots (duration $250 \mathrm{~ms}$; ISI $1.2 \mathrm{~s}$ ) at randomly interleaved azimuthal (elevational) locations, with the elevation (azimuth) held constant at the best value for the site. The direction of motion of the dot was vertical (horizontal) for azimuthal (elevational) tuning curves. The azimuthal and elevational tuning curves that we report here were measured in a subsequent set of trials, consisting of single looming dots interleaved with two-stimulus competition trials.

Looming dots. Looming visual stimuli consisted of a black dot on a gray background that progressively increased in size over the period of stimulus presentation $(250 \mathrm{~ms})$ starting from a size of $0.6^{\circ}$ radius. The center of a looming stimulus remained constant throughout the duration of the motion stimulus (unlike translationally moving stimuli). The rate of change of the angular size of the dot, $d \theta / d t$, where $\theta$ is the visual half-
A Looming dot

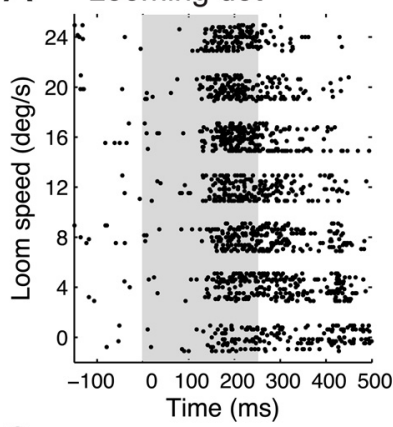

$\mathrm{C}$

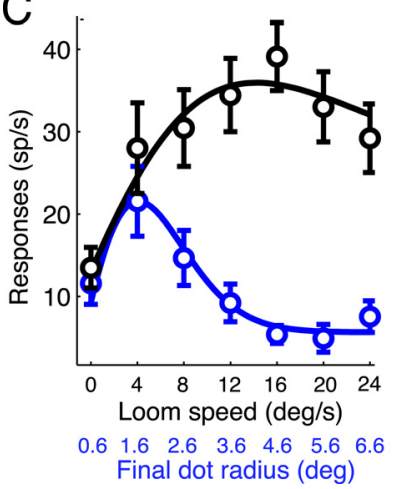

B Stationary dot

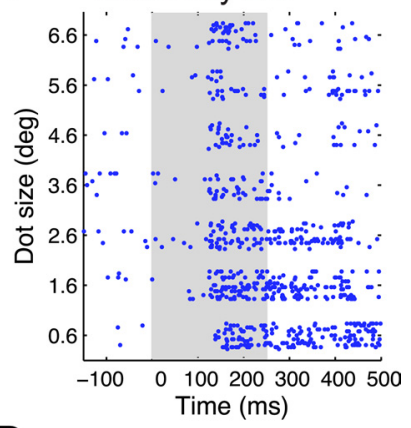

D

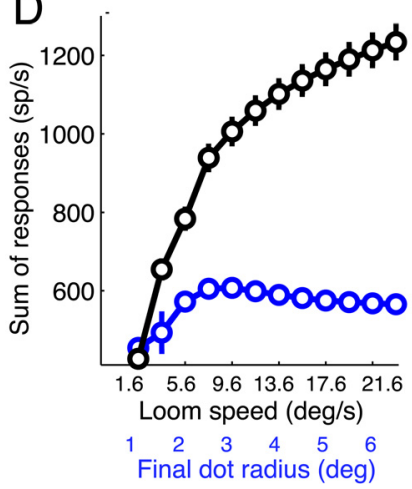

Figure 1. Neuronal responses to looming and stationary dots. $\boldsymbol{A}$, Raster display of OTi-d neuronal responses to looming, black dots of different loom speeds. The duration of the stimulus, indicated by the gray box, was $0-250 \mathrm{~ms}$. $\boldsymbol{B}$, Raster display of responses of the same unit to stationary, black dots of different sizes (radii). The sizes tested correspond to the final dot sizes for each of the loom speeds tested in $A$. The duration of the stimulus, indicated by the gray box, was $0-250 \mathrm{~ms}$. C, Neuronal responses to loom speed and dot size (black and blue circles, respectively, shown as mean \pm SEM) obtained from $\boldsymbol{A}$ and $\boldsymbol{B}$ by counting spikes from 0 to 400 $\mathrm{ms}$ after stimulus onset and converting to spikes per second. The curves are difference of Gaussian models that fit best to the data (see Materials and Methods). Loom speeds and the corresponding final dot sizes are indicated along the $x$-axis. The maximum suppression (response at largest dot size or loom speed/peak response) in both curves is at the 10-percentile value of the respective distributions of maximum suppression ( $n=19$ sites). $\boldsymbol{D}$, Total number of spikes (per second) in response to looming dots (black curve) and stationary dots (blue curve), calculated from a population of 19 sites at which both types of stimuli were tested. The error bars indicate the SE, estimated by applying a bootstrapping procedure with 1000 resamplings.

angle subtended by the object at the eye, was held constant over the duration of stimulus presentation $(d \theta / d t=L)$, leading to a linear relationship between dot size and time. The constant $L$ was denoted as the loom speed of the stimulus in degrees per second. Different loom speeds were achieved by changing the final size of the dot, while keeping the initial size $\left(0.6^{\circ}\right)$ and the duration of presentation $(250 \mathrm{~ms})$ fixed. Looming stimuli with a nonlinear relationship of dot-size as a function of time (mimicking a constant approach speed) were also tried, but were not used for data collection. At moderately high approach speeds, these stimuli resulted in final dot-sizes that were as large or larger than the sizes of the visual RFs, making the study of stimulus integration within the visual RF difficult.

Habituation. Responses of neurons in the OTi-d habituated to the repetition of any stimulus (light, dark, moving, or stationary dots). To minimize the effects of habituation, we used long interstimulus intervals (up to $5.0 \mathrm{~s}$ ), looming instead of stationary dots, and we jittered the location of stimuli on subsequent presentations when locations were sampled repeatedly. Stimulus locations were jittered within a small region of the desired, center location. For stimuli presented inside the RF, nine jitter positions were used: one at a central position and eight at randomly chosen positions along the circumference of a circle of radius $1^{\circ}$ around the central position. For stimuli presented outside the RF, nine jitter positions were used: one at a central position and eight along the edges of a rectangle measuring $2-4^{\circ}$ by $6-8^{\circ}$, centered on the central position. The smaller edge of the rectangle was parallel to the axis along 
A
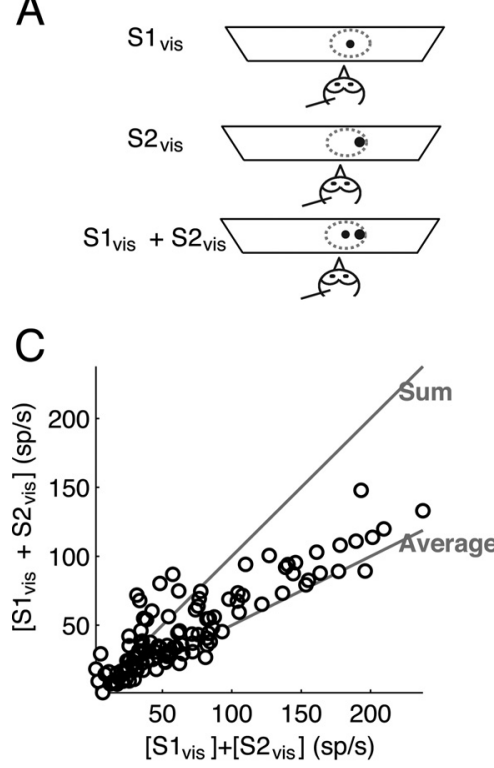

Figure 2. Responses to paired looming visual stimuli inside the RF. A, Schematic representation of the experimental set-up showing (in top-view) an owl, a recording electrode, the tangent screen, the visual RF of the site (dotted circle). One visual stimulus $\left(S 1_{\text {vis }}\right.$ small black dot) was a looming stimulus presented at the RF center, and the second ( $S 2_{\text {vis, }}$ larger black dot) was a faster looming visual stimulus presented inside the RF such that it did not physically overlap with $S 1_{\text {vis }}$. B. Distribution of the ratio of responses to two looming visual stimuli $\left(S 1_{\text {vis }}\right.$ and $\left.S 2_{\text {vis }}\right)$ presented simultaneously inside the $R F$ to the sum of the responses to each stimulus presented alone for all paired tests ( $n=30$ sites; 118 paired-stimulus tests). The properties of $S 1_{\text {vis }}$ and $S 2_{\text {vis }}$ are given in the text. C, Responses to the two looming visual stimuli presented simultaneously plotted against the sum of the responses to each stimulus alone. The data indicate mean values. The lines along which the data points would fall if the responses to $\mathrm{S}_{\text {vis }}$ and $\mathrm{S} 2_{\text {vis }}$ were equal to either the sum or the average of the individual responses are indicated. $\boldsymbol{D}$, Responses to the two looming visual stimuli presented simultaneously plotted against the responses to $S 1_{\text {vis }}$ alone. The line along which the data points would fall if the responses to $\mathrm{S}_{\text {vis }}$ and $\mathrm{S} 2_{\text {vis }}$ were equal to the responses to $S 1_{\text {vis }}$ alone is indicated.

which a tuning curve was being measured. For instance, when the azimuthal position of a competitor stimulus was varied, the rectangle of jitter was $2-4^{\circ}$ in azimuth and $6-8^{\circ}$ in elevation.

\section{Auditory stimulation}

Stimuli. Auditory stimuli were generated using customized MATLAB software interfaced with Tucker Davis Technologies hardware (RP2). Sounds were filtered with head-related transfer functions (HRTFs) from a typical barn owl (Witten, 2008) and delivered binaurally through matched earphones (ED-1914; Knowles Electronics) coupled to damping assemblies (BF-1743) inserted into the ear canals $\sim 5 \mathrm{~mm}$ from the eardrums. The amplitudes of the two earphones were equalized to within $\pm 2 \mathrm{~dB}$.

Spatial tuning curves. Spatial tuning to auditory stimuli was measured using noise bursts $(250 \mathrm{~ms}$ duration, $2-10 \mathrm{kHz}, 0 \mathrm{~ms}$ rise/fall times, $10-20 \mathrm{~dB}$ above unit threshold, ISI $=1.2 \mathrm{~s}$ ). Tuning to azimuth (elevation) was assessed by presenting 10-15 series of HRTF-filtered noise bursts for randomly interleaved azimuthal (elevational) locations while holding the elevation (azimuth) constant at the best value for the site.

\section{Two-stimulus measurements}

Stimuli are referred to as S1 and S2, with subscripts indicating whether they were visual or auditory. The $S 1_{\text {vis }}$ stimulus was always a visual looming stimulus with a loom speed that evoked $55 \pm 6 \%$ of the maximum loom response at that site, referred to as the standard looming stimulus. The $S 2_{\text {vis }}$ stimulus was also always a visual looming stimulus with a speed that was greater than that of $S 1_{\text {vis }}$. On average, the $S 2_{\text {vis }}$ stimulus evoked a response that was $125 \pm 6 \%$ of the response to $S 1_{\text {vis }}$ (when each was presented at the center of the RF). The $\mathrm{S}_{2}$ aud stimulus was a broadband noise burst $10-20 \mathrm{~dB}$ above unit threshold. Only sites that responded to the $S 2_{\text {aud }}$ stimulus presented alone were tested for cross-modal interactions. On average, the response to $\mathrm{S} 2_{\text {aud }}$ presented at the center of the RF was $48 \pm 7 \%$ of the response to $S 1_{\text {vis. }}$. The centers of the visual RFs of the sites at which the competition experiments were performed were distributed in the azimuth from ipsilateral $3.5^{\circ}$ to contralateral $43^{\circ}$ and in the elevation from $-43^{\circ}$ to $+40^{\circ}$.

Visual stimuli could be presented over the range of $\pm 45^{\circ}$ in azimuth and from $-50^{\circ}$ to $+40^{\circ}$ in elevation. Consequently, in twostimulus experiments, the maximum distance between the $S 1_{\text {vis }}$ and $S 2_{\text {aud }}$ stimuli could range up $90^{\circ}$. The maximum distance between $S 1_{\text {vis }}$ and $\mathrm{S} 2$ aud that was tested was $60^{\circ}$.

Two stimulus-interactions inside the RF were studied by presenting $\mathrm{S}_{\text {vis }}$ at the center of the RF, and $\mathrm{S} 2$ at two off-center locations along the azimuthal axis (one on each side of the center) and at 2-4 off-center locations along the elevational axis (1-2 locations on each side of the center). When the S2 stimulus was visual, its locations inside the RF were chosen to ensure that there was no overlap between $S 1_{\text {vis }}$ and $S 2_{\text {vis }}$ at any time during the presentation period.

\section{Data analysis}

Net responses at each OTi-d site were quantified by subtracting the average firing rate that occurred during the prestimulus period across all interleaved trials (baseline activity) from the average firing rate that occurred during a fixed window after stimulus onset. In Figure 1, a window of $0-400 \mathrm{~ms}$ was used. In all competition experiments, responses to single visual stimuli or to two visual stimuli were obtained using a window of 100 to $250 \mathrm{~ms}$ after stimulus onset. In cases where an auditory stimulus was presented along with a visual stimulus, responses were obtained using a window of 150 to $250 \mathrm{~ms}$ after stimulus onset. The Lilliefors test (lillietest in MATLAB) was used to determine whether datasets were normally distributed. When the assumptions of normality were met, $t$ tests (ttest in MATLAB) were used for comparisons, and data were summarized as mean \pm SEM. When normality assumptions were not met, non-parametric sign-tests (signtest in MATLAB) were used for comparisons, and data were summarized by the median along with $95 \%$ confidence intervals, reported as "median [low, high]".

Spatial RFs. The visual RF was defined for each site as the portion of the visual field wherein a single visual stimulus evoked responses above baseline, based on azimuthal and elevational tuning curves. Similarly, the auditory RF was defined for each site as the set of locations at which a single auditory stimulus evoked responses above baseline. A location was deemed to be inside the RF if baseline-subtracted responses to the stimulus alone at that location were significantly greater than zero. Statistical significance $(p<0.05)$ was determined by applying an ANOVA procedure (anoval in MATLAB) followed by a correction for multiple comparisons (multcompare in MATLAB). The center of the RF was defined as the center of a unidimensional Gaussian fit to azimuthal and elevational tuning curves. The half-max widths of the RFs were determined as the widths of the Gaussian fits at half of the maximal response.

Responses to looming dots. Responses to looming dots (Fig. 1) were fit with three different models: sigmoidal (Eq. 1), linear, and difference of Gaussians (Eq. 2), using the MATLAB command nlinfit.

$$
R(x)=c+\frac{s}{\left(1+e^{-m(x-d)}\right)},
$$

where $R(x)$ is the baseline-subtracted response as a function of the loom speed of the dot, $c$ is the minimum response, $s$ is the maximum response, $d$ is the loom-speed at which the response is mid-way between the minimum and the maximum, and $m$ is the slope parameter. 
The model that produced the lowest Akaike Information Criterion (Akaike, 1974), i.e., the model that produced the smallest sum of squared residuals with the least number of parameters, was chosen as the best fit for the data for that site. The responses to stationary dots rose to a peak value and then declined asymptotically to a steady-state value, following the classic difference-of-Gaussians response profile. Hence responses to stationary dots were fit just with the difference-ofGaussians model.

The time course of responses to looming dots (see Fig. $9 B-D$ ) was characterized by estimating instantaneous firing rates (IFRs) by smoothing the PSTH from each trial (bin size 5 $\mathrm{ms})$ with a Gaussian filter ( $\sigma=25 \mathrm{~ms})$, and determining the mean (and SEM) across the trials. Response latency (in ms) was defined as the time at which the mean IFR exceeded the baseline firing rate by 1.96 times the baseline SD. The peak response and time to peak were determined, respectively, as the maximum of the mean IFR and the time at which this maximum occurred. Responses corresponding to stimulus offset $\left(r^{\text {off }}\right)$ were determined as the mean IFR at the time $t=250+$ latency, where $250 \mathrm{~ms}$ is the duration of stimulus presentation and latency is the response latency. As a function of loom speed, $r^{\text {off }}$ was well accounted for by a difference of Gaussians model (see Fig. 9D, $\left.r_{\text {off }}\right)$. The resulting fit was used to determine the steady-state value, $r_{\text {off }}^{\text {ss }}$.

Difference of Gaussians model. Responses to stationary dots of increasing size (dot sizeresponse function) exhibited a classic behavior (Fig. $1 B$, blue) and were analyzed using a standard technique from retinal and cortical physiology (Rodieck, 1965; DeAngelis et al., 1994; Sceniak et al., 1998) that fits a difference of Gaussians model to responses. This model assumes that the excitatory and inhibitory influences are each Gaussians centered on the classical RF, and that they sum linearly to yield the net response to a stimulus, as follows:

$$
R(s)=R_{0}+K_{e} \int_{-s}^{s} e^{-(2 y / a)^{2}} d y-K_{i} \int_{-s}^{s} e^{-(2 y / b)^{2}} d y,
$$

where, $R(\mathrm{~s})$ is the response as a function of the radius of the dot, $R_{0}$ is the baseline activity, $K_{\mathrm{e}}$ and $K_{\mathrm{i}}$ are constants that determine the relative strengths of the excitatory and inhibitory Gaussians, respectively, and $a$ and $b$ are the excitatory and inhibitory space constants, respectively. The size of the classical inhibitory surround was estimated as the dot-radius at which the response reached $5 \%$ of the steady-state value.

Responses to two stimuli. To assess the competitive effect of a second, discrete stimulus outside the $\mathrm{RF}$ ( $\mathrm{S} 2_{\text {vis }}$ or $\mathrm{S} 2_{\text {aud }}$ ) on spatial tuning curves measured with a visual stimulus (see Figs. $3,7, S 1_{\text {vis }}$ ), we analyzed the relationship between the responses to the two stimuli presented together to the response to the $\mathrm{S1}_{\text {vis }}$ stimulus alone (see Figs. 3, 7), using the robust linear regression function (robustfit in MATLAB) to fit a line to the data pairs. This function uses an iteratively reweighted least-squares algorithm and is less sensitive to outliers than is ordinary regression. A fit was deemed to be good if the residuals were distributed normally. The intercept parameter was considered to be significantly different from zero if the $p$ value associated with the null hypothesis that it was distributed with a mean of zero (one of the outputs of the function) was $<0.05$. The slope parameter was considered to be significantly different from 1 at a significance level of 0.05 when the $95 \%$ confidence interval of its estimate did not overlap with 1 .

To assess the competitive effect of a second stimulus ( $\mathrm{S} 2 \mathrm{vis}_{\text {is }}$ or $\mathrm{S} 2{ }_{\text {aud }}$, respectively) located outside the $\mathrm{RF}$ on responses to a visual stimulus $\left(\mathrm{S}_{\mathrm{vis}}\right)$ centered in the visual RF (see Figs. 4, 8A,B), we calculated the percentage-change in the response to two stimuli $\left(\left[S 1_{\text {vis }}+S 2_{\text {vis }}\right]\right.$ or $\left.\left[S 1_{\text {vis }}+S 2_{\text {aud }}\right]\right)$ with respect to the responses to the $S 1_{\text {vis }}$ stimulus alone. To determine those locations at which the S2stimulus had a significant effect $(p<0.05)$, we performed a one-way ANOVA (anova1 in MATLAB) followed by a multiple comparison correction (multcompare in MATLAB) on the percentage-change response distributions. Percentage changes in response that were not significantly different from zero are shown as gray dots in Figures 5 and $8 C$. The population summary data (open circles) were obtained by binning locations into bins of $10^{\circ}$ and $15^{\circ}$ for azimuth and elevation, respectively. The average percentage suppression within each bin was plotted as mean \pm SEM, against the bin center, computed as the average of the locations within the bin and shown along with its SEM.

For the above analyses, a location was deemed to be inside the RF if the presentation of the $\mathrm{S} 2$ stimulus alone at that location elicited greater than baseline activity, and otherwise, outside. 

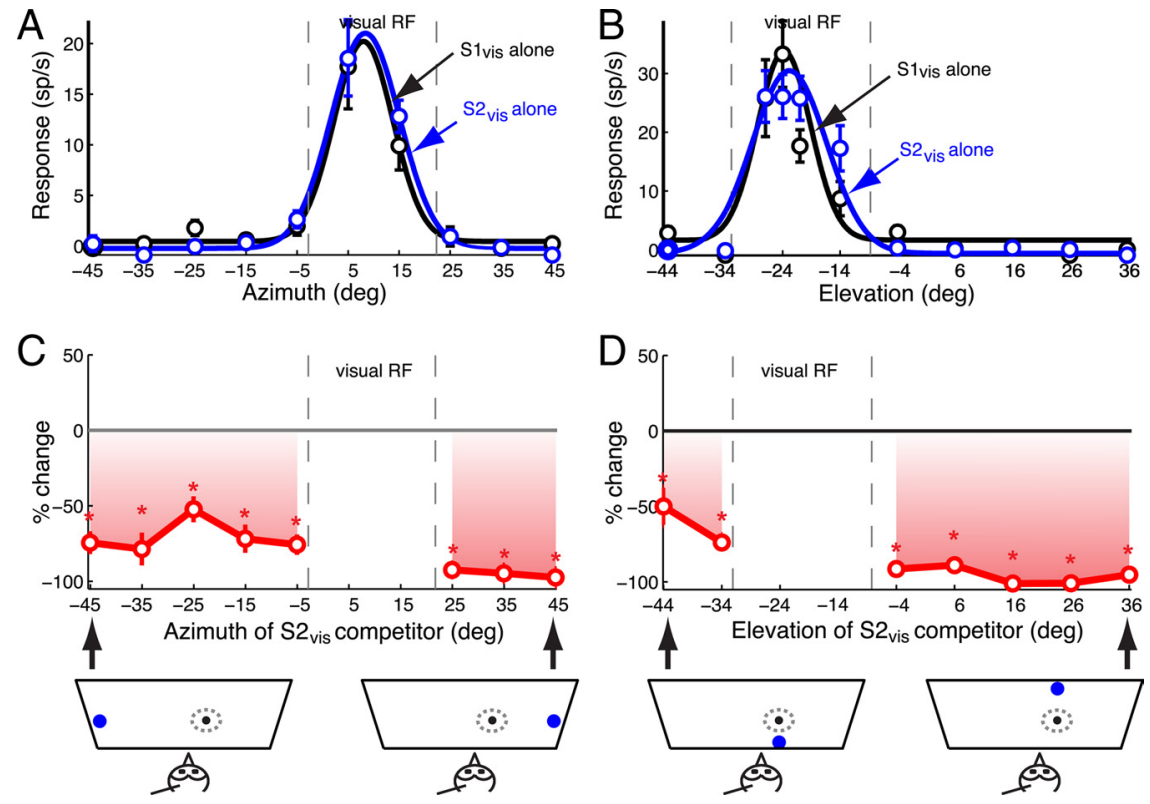

Figure 4. Spatial profile of the effect of a visual competitor on responses to a visual stimulus centered in the RF. A, Azimuthal tuning: responses and best-fit Gaussian profiles of an $0 \mathrm{Ti}-\mathrm{d}$ site to single looming stimuli presented at different azimuths. $\mathrm{S} 1_{\text {vis }}$ (loom speed $=8 \% \mathrm{~s}$, black) and $\mathrm{SZ}_{\text {vis }}$ (speed $=14 \% \mathrm{~s}$, blue). Positive (negative) azimuths represent locations contralateral (ipsilateral) with respect to the recording site. The data indicate mean \pm SEM. $\boldsymbol{B}$, Elevational tuning: responses and best-fit Gaussian profiles for the same site as in $A$. Positive (negative) elevations represent locations above (below) the visual plane. C, Top, Azimuth-response profile for paired looming stimuli. Site exhibited a pronounced effect in the presence of $S 2_{\text {vis }}$. Responses to $S 1_{\text {vis }}$ and $S Z_{\text {vis }}$ presented simultaneously as percentage changes (mean $\pm S E M$ ) with respect to the responses to the $S 1_{\text {vis }}$ stimulus presented alone. $\mathrm{S1}_{\text {vis }}$ was presented at the RF center, while $\mathrm{SZ}_{\text {vis }}$ was presented at various azimuths outside the RF. Asterisks indicate significant response suppression ( $p<0.05$, see Materials and Methods). Bottom, Schematic diagram showing the stimulus configuration for two extreme $S 2_{\text {vis }}$ locations. $\boldsymbol{D}$, Top, Elevation-response profile for paired looming stimuli. Site exhibited a pronounced effect in the presence of $\mathrm{S2}_{\text {vis. }}$. Same conventions as in $\boldsymbol{C}$.

\section{Results}

\section{Responses to looming stimuli}

Stimulus competition in the OTi-d was studied with looming dots as the standard stimulus. Visual RF centers ranged from ipsilateral $3.5^{\circ}$ to contralateral $43^{\circ}$ in azimuth, and from $-43^{\circ}$ to $+40^{\circ}$ in elevation. The median size of the visual RFs, measured as the distance from the visual RF center to its edge, was $5.3^{\circ}$ in azimuth and $8.7^{\circ}$ in elevation $(n=28$, and 22 sites, respectively).

Looming dots were extremely effective in driving sustained responses. Neuronal responses increased with loom speed. For the site illustrated in Figure $1 A$, as loom speed increased beyond $12 \%$, the duration of the responses became shorter, indicating the engagement of a local inhibitory surround, an issue that will be addressed at the end of Results. Summed across all sites, responses increased with loom speed for speeds up to at least $20 \%$ (Fig. $1 D$, black curve).

Faster loom speeds were always accompanied by larger final dot sizes (see Materials and Methods). The increased response to faster loom speeds was not due to the larger final dot sizes, however. OTi-d neurons preferred small dot sizes when tested with stationary dots (Fig. 1C,D; blue curves). Moreover, responses to looming stimuli were much stronger than responses to stationary dots of identical final dot sizes (Fig. 1). These data demonstrate that the speed of the looming stimulus, rather than the final size of the dot, was the primary determinant of neural responses in the OTi-d.

\section{Responses to paired visual stimuli inside the RF}

Neuronal responses to a looming visual stimulus located inside the RF were affected differently by a second looming visual stimulus, depending critically on whether the second visual stimulus was inside or outside the RF. To explore this effect quantitatively, we first measured neuronal responses with two simultaneous looming visual stimuli, both located inside the RF, and compared them to the responses obtained to each stimulus presented individually (Fig. 2A). For these experiments, one stimulus $\left(\mathrm{S}_{\text {vis }}\right)$ was the standard looming stimulus (see Materials and Methods) and was located at the RF center (Fig. 2A). The second stimulus $\left(\mathrm{S} 2_{\mathrm{vis}}\right)$ was a dot that loomed as fast or faster than the $S 1_{\text {vis }}$ stimulus and was presented at 2-6 locations inside the RF such that there was no overlap between the two stimuli (Fig. 2 A) (see Materials and Methods), leading to a total of 118 paired tests from 30 sites. The median ratio of the responses to $\mathrm{S} 2$ vis alone to the responses to $\mathrm{S} 1_{\text {vis }}$ alone was 1.19 , indicating that, on average, $\mathrm{S} 2{ }_{\text {vis }}$ (presented at the various off-center locations) was $119 \%$ as effective at driving responses as was $S 1_{\text {vis }}$ (located at the RF center).

Responses to $\mathrm{S}_{\text {vis }}$ and $\mathrm{S} 2_{\text {vis }}$ presented simultaneously inside the RF varied across recording sites and across locations inside the RF (Fig. $2 B, C$ ). The range of observed effects was quantified using the ratio of the responses elicited when $S 1_{\text {vis }}$ and $S 2$ vis were presented together over the sum of the responses when they were presented individually ([S1 $1_{\mathrm{vis}}+$ $\left.\mathrm{S} 2_{\mathrm{vis}}\right] /\left[\mathrm{S}_{\mathrm{vis}}\right]+\left[\mathrm{S} 2_{\mathrm{vis}}\right]$, Fig. $\left.2 \mathrm{~B}\right)$; responses to $\mathrm{S} 2_{\mathrm{vis}}$ alone were included in the denominator because $\mathrm{S} 2{ }_{\mathrm{vis}}$, when presented inside the RF, drove responses. This ratio ranged from 0.25 (subadditive) to 1.15 (superadditive). Across a population of 30 sites, the predominant effect was a subadditive ratio of $0.62 \pm 0.04(n=$ 118), close to an averaging of the responses to each stimulus alone (ratio of 0.5 ), consistent with response averaging observed in previous studies in the mammalian SCi (Li and Basso, 2005; Alvarado et al., 2007) and primate visual cortices (Reynolds et al., 1999). The integration ratio was not uniformly distributed across the magnitudes of responses to each stimulus alone (Fig. 2C). When responses to the individual stimuli were weak, responses to the paired stimuli ranged from subadditive to superadditive, whereas when responses to individual stimuli were strong, responses to the paired stimuli were subadditive and close to an average of the individual responses. Paired-stimulus responses were also plotted against the responses to $\mathrm{S1}_{\text {vis }}$ alone (Fig. $2 \mathrm{D}$ ). When plotted this way, the paired responses almost always showed an enhancement over the responses to $S 1_{\text {vis }}$ alone, consistent with an averaging rule, since $\mathrm{S} 2$ vis generally evoked stronger responses than $\mathrm{S1}_{\text {vis }}$ when presented alone.

\section{Effect of a distant visual competitor on visual tuning curves}

The effect of a second visual stimulus on neuronal responses changed dramatically when the second stimulus was located outside the RF. In these experiments, neuronal responses were driven with a standard $\mathrm{S}_{\text {vis }}$ looming stimulus presented at various azimuths (or elevations) across the RF while another looming stimulus, $\mathrm{S} 2_{\text {vis }}$, which was at least as strong as $\mathrm{S}_{\text {vis }}$, was presented 
outside the RF at a location $30^{\circ}$ lateral to the RF center. Under these conditions, the $\mathrm{S} 2_{\text {vis }}$ stimulus usually suppressed neuronal responses to the $S 1_{\text {vis }}$ stimulus. Therefore, for describing these results, we refer to $\mathrm{S} 2$ vis as the "competitor stimulus."

The competitor stimulus typically exerted a potent divisive influence on responses to the $S 1_{\text {vis }}$ stimulus without altering the spatial tuning of the site. An example is shown in Figure $3 A-D$. Responses to $S 1_{\text {vis }}$ presented alone were strong and sustained (Fig. 3A). In contrast, when the competitor stimulus was presented on interleaved trials far lateral to the RF center ( $30^{\circ}$ away), responses to $\mathrm{S} 1_{\text {vis }}$ were severely suppressed (Fig. $3 B, C$ ). By plotting the responses to the paired $S 1_{\text {vis }}$ and $S 2_{\text {vis }}$ stimuli as a function of responses to the $S 1_{\text {vis }}$ stimulus alone, we observed a linear relationship (Fig. $3 D, R^{2}=0.8$ ). The slope of the line (slope = 0.57 ) indicated that the competitor stimulus exerted a strong divisive influence on responses to the $S 1_{\text {vis }}$ stimulus alone. The $y$-intercept of this relationship was not significantly different from zero, indicating no subtractive influence.

Measurements were made following this protocol on a population of 25 sites (Fig. 3E). Across the population, the slope of the linear fit to the normalized data was 0.50 , indicating a potent divisive influence. The $y$-intercept was $7 \%$ of the maximum firing rate, reflecting a small additive enhancement of weak responses by the $\mathrm{S} 2_{\text {vis }}$ stimulus. Consistent with a primarily divisive effect, there was no change in either visual RF centers or widths of spatial tuning ( $p>0.05$, paired $t$ tests). Thus, a distant visual competitor exerted a powerful divisive influence on spatial tuning curves in the OTi-d.

\section{Spatial profile of visual stimulus competition}

We next explored the dependence of stimulus competition on the spatial location of the second visual stimulus, $\mathrm{S} 2$ vis. For these measurements, the $S 1_{\text {vis }}$ stimulus was always the standard looming stimulus, presented near the center of the RF, and jittered to minimize adaptation (see Materials and Methods). On interleaved trials, a competitor stimulus ( $\mathrm{S} 2_{\text {vis }}$ ), which was at least as strong as $S 1_{\text {vis }}$, was presented simultaneously outside of the RF at various locations in azimuth and elevation (see Materials and Methods). Spatial profiles of stimulus competition were obtained by plotting responses to the paired $\mathrm{S} 1_{\text {vis }}$ and $\mathrm{S} 2_{\text {vis }}$ stimuli as a percentage change with respect to the responses to the $S 1_{\text {vis }}$ stimulus alone for different locations of the competing S2 $2_{\text {vis }}$ stimulus.

Spatial profiles of visual stimulus competition, measured at a site for which the $\mathrm{S}_{\text {vis }}$ stimulus had a pronounced effect, are shown in Figure 4. Responses to either the $\mathrm{S}_{\text {vis }}$ or $\mathrm{S} 2_{\text {vis }}$ stimulus alone were strong and similarly tuned both in azimuth (Fig. 4A) and in elevation (Fig. $4 B$ ) at this site (and across all sites; $p>0.05$, paired $t$ tests). On interleaved trials, when $S 1_{\text {vis }}$ and the competitor $S 2_{\text {vis }}$ stimulus were presented together, the competitor stimulus suppressed responses to $\mathrm{S} 1_{\text {vis }}$ from all locations, in azimuth and elevation, outside of the RF (Fig. 4C,D). For some locations of the competitor stimulus, the suppression of responses to $S 1_{\text {vis }}$ was complete (no spikes; $-100 \%$ ). The suppressive effect was potent for competitor stimulus locations above and below the RF, as well as to the right and left of the RF. The suppressive effects for competitor stimulus locations on the far left (Fig. 4C, left side; the hemifield opposite to the one containing the RF) are remarkable, considering that locations beyond $15^{\circ}$ left are not represented on the same side of the brain; the map of space in the OT represents the contralateral hemifield and $\sim 15^{\circ}$ into the ipsilateral hemifield (Knudsen, 1982). Thus, this suppression must have been caused by inhibitory influences driven from the opposite side of the brain.
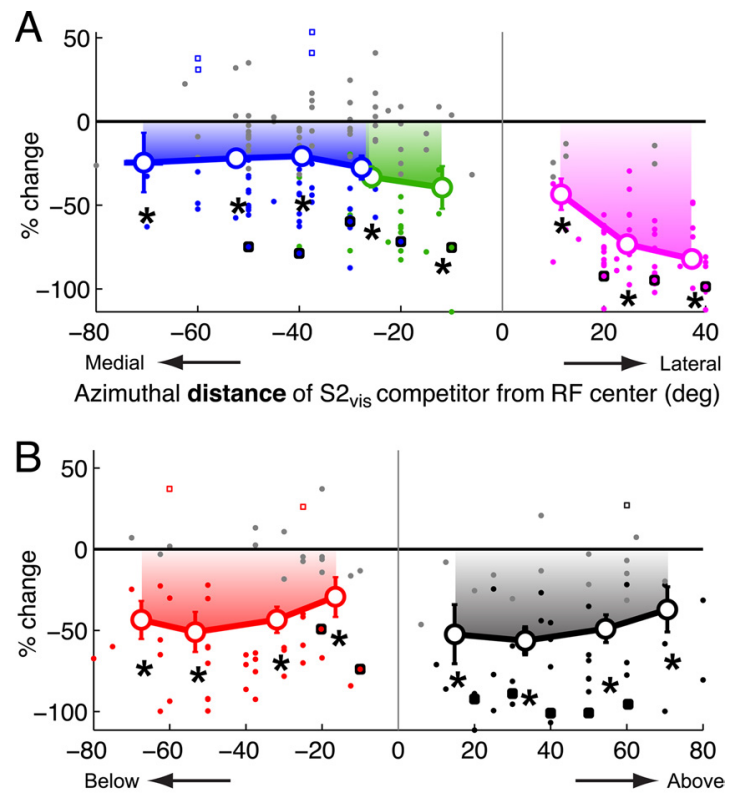

Elevational distance of $\mathrm{S} 2_{\text {vis }}$ competitor from RF center (deg)

Figure 5. Population summary of the spatial profile of the effect of a visual competitor on responses to a visual stimulus centered in the RF. $A$, Summary of the effect of the azimuthal position of a looming visual stimulus $\left(S_{2}\right.$ vis $)$ located outside the $\mathrm{RF}$ on responses to a weaker looming visual stimulus $\left(S 1_{\text {vis }}\right)$ presented near the RF center $(n=33)$. Responses are shown as percentage changes (mean $\pm \mathrm{SEM}$ ) with respect to the responses to $S 1_{\text {vis }}$ alone, and are plotted as a function of the azimuthal distance of $S 2_{\text {vis }}$ from the RF center. Only locations that were outside the RF (i.e., locations at which single stimuli did not drive responses; Materials and Methods) were included. Positive values of distance represent locations lateral to the RF center; negative values represent locations medial to the RF. Filled dots indicate data from individual sites; large open circles indicate the average effect (see Materials and Methods); gray dots indicate individual data that were not significantly different from 0 (see Materials and Methods); squares with black borders indicate the data from the example site in Figure 4. Locations lateral to the RF are shown in pink, those medial to the RF center and up to $15^{\circ}$ into the opposite hemifield (represented on the same side of the brain) are shown in green, locations $>15^{\circ}$ into the opposite hemifield (represented on the opposite side of the brain) are shown in blue. Asterisks indicate significant response suppression ( $p<0.05$, see Materials and Methods). $\boldsymbol{B}$, The effect of the elevational position of a looming visual stimulus $\left(S 2_{\text {vis }}\right)$ located outside the $\mathrm{RF}$ on responses to a weaker looming visual stimulus $\left(S 1_{\text {vis }}\right)$ presented near the RF center $(n=$ 24). Same conventions as in $\boldsymbol{A}$. Positive (negative) values of distance represent locations above (below) the RF center. Asterisks indicate significant response suppression ( $p<0.05$, see Materials and Methods).

Spatial profiles of visual stimulus competition were measured following this protocol for $33 \mathrm{OTi}$-d sites. Of these, three sites exhibited no competitor modulation of responses to the $S 1_{\text {vis }}$ stimulus under these conditions. Of the remaining $30(91 \%)$ sites, 24 were tested with competitors in both azimuth and elevation, and six were tested only in azimuth. The responses were plotted with competitor locations expressed as distance from the RF center, allowing the data to be pooled across sites. Responses were analyzed separately for five different regions of space: locations lateral to the RF (Fig. $5 A$, pink), locations medial to the RF and up to $15^{\circ}$ into the opposite hemifield (Fig. $5 A$, green), locations medial to the RF and $>15^{\circ}$ into the opposite hemifield (represented on the opposite side of the brain) (Fig. 5A, blue), locations above the RF (Fig. $5 B$, black), and locations below the RF (Fig. 5B, red).

The locations of the competitor stimulus that were consistently most effective in inhibiting responses to the $\mathrm{S}_{\text {vis }}$ stimulus were far lateral to the RF (Fig. $5 A$, pink, points $\geq 20^{\circ}$ away). When the competitor was $>20^{\circ}$ away from the center of the RF on the contralateral side, response suppression averaged $76 \pm 3 \%(p<$ 
A
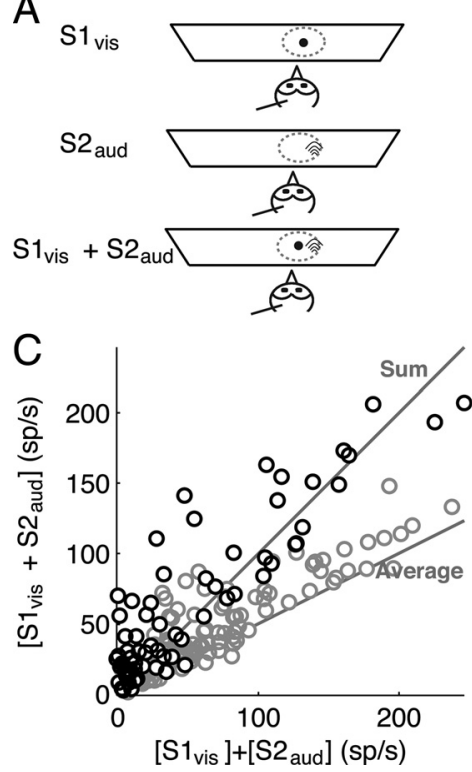

B

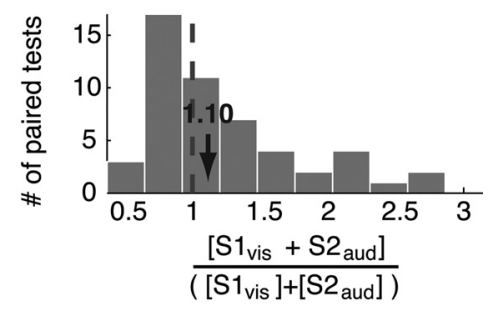

$\mathrm{D}$

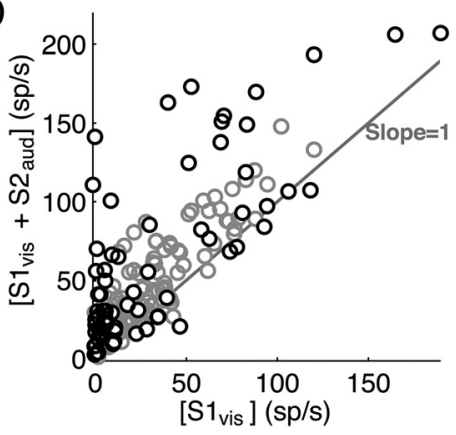

Figure 6. Responses to paired visual and auditory stimuli inside the RF. $A$, Schematic representation of the experimental set-up. $S 1_{\text {vis }}$ (small black dot) was a looming stimulus presented at the $R F$ center, and $S 2_{\text {aud }}$ (black arcs) was an auditory stimulus presented inside the auditory RF. $\boldsymbol{B}$, Distribution of the ratio of responses to a pair of visual $\left(S 1_{\text {vis }}\right)$ and auditory stimuli $\left(S 2_{\text {aud }}\right)$ presented simultaneously inside the RF to the sum of the responses to each stimulus presented alone for all paired tests ( $n=14$ sites; 69 paired-stimulus tests). The properties of $S 1_{\text {vis }}$ and $S 2_{\text {aud }}$ are given in the text. $C$, Responses to the paired visual ( $\left.S 1_{\text {vis }}\right)$ and auditory stimuli ( $\left(\mathrm{S}_{\mathrm{aud}}\right)$ plotted against the sum of the responses to each stimulus alone. The data indicate mean values. The lines along which the data points would fall if the responses to $\left[\mathrm{S}_{\text {vis }}+\mathrm{SZ}_{\text {aud }}\right.$ ] were equal to either the sum or the average of the individual responses are indicated. Responses to two visual stimuli presented inside the visual RF (Fig. 2C) are reproduced for comparison (gray circles). $\boldsymbol{D}$, Responses to the paired visual $\left(S 1_{\text {vis }}\right)$ and auditory stimuli $\left(S 2_{\text {aud }}\right)$ presented simultaneously plotted against the responses to $S 1_{\text {vis }}$ alone. The line along which the data points would fall if the responses to $S 1_{\text {vis }}$ and $S 2_{\text {aud }}$ were equal to the responses to $S 1_{\text {vis }}$ alone is indicated. Responses to two visual stimuli presented inside the visual RF (Fig. 2D) are reproduced for comparison (gray circles).

$\left.10^{-4}\right)$. As the competitor approached within $20^{\circ}$ of the RF center on the lateral side, the average suppression decreased to $43.5 \pm$ $9.2 \%$ ( $p=0.002 ; t$ test $)$. For competitor locations that were medial to the RF and were represented on the same side of the brain as the RF (Fig. $5 A$, green), the average suppression was 35\% $\pm 5.6 \%(p<$ $10^{-6}$, $t$ test). Competitor locations above (Fig. $5 B$, black) or below (Fig. $5 B$, red) the RF were, on average, equally effective in suppressing responses to $S 1_{\text {vis }}$; the average response suppression across both of these regions was $46.4 \pm 3.8 \%$. Competitor locations that induced the weakest response suppressions were those located medial to the RF and with azimuths $>15^{\circ}$ into the opposite hemifield (Fig. 5A, blue). Response suppression by a competitor located in this region averaged 23\% $\pm 3.3 \%\left(p<10^{-7}\right.$, $t$ test). In rare instances ( $<3 \% ; 7 / 288$ measurements), the visual competitor facilitated the responses to the $\mathrm{S}_{\text {vis }}$ stimulus under these conditions (Fig. 5, open squares; $p<0.05$ ).

\section{Responses to paired visual and auditory stimuli inside the excitatory RF}

Previous research has shown that most neurons in the owl's OTi-d respond both to auditory and to visual stimuli, and the centers of the spatial RFs for auditory and visual stimuli are mutually aligned (Knudsen, 1982). We measured responses of OTi-d sites to the paired presentation of auditory and visual stimuli, when both stimuli were located inside their RFs. For these experiments, the auditory stimulus ( $\left.\mathrm{S} 2_{\text {aud }}\right)$ was a $250 \mathrm{~ms}$ broadband noise burst, $10-20 \mathrm{~dB}$ above threshold, presented in virtual space (see Materials and Methods) and $S 1_{\text {vis }}$ was, again, the standard looming stimulus (Fig. $6 \mathrm{~A}$ ). $\mathrm{S} 1_{\text {vis }}$ was always presented at the $\mathrm{RF}$ center, while $S 2_{\text {aud }}$ was presented at 2-6 off-center locations inside the auditory RF (Fig. 6A) (see Materials and Methods). Single visual and auditory and paired bimodal stimulus presentations were interleaved. Data were collected for 14 sites (a total of 69 paired stimulus tests); every site tested was responsive to $\mathrm{S} 2$ aud presented alone. The median ratio of the responses to $S 2_{\text {aud }}$ alone to the responses to $S 1_{\text {vis }}$ alone was 0.35 , indicating that, on average, the $\mathrm{S} 2$ aud stimulus (presented at the various off-center locations) was $35 \%$ as effective at driving responses as was the $\mathrm{S}_{\text {vis }}$ stimulus (located at the RF center).

Responses to the bimodal stimuli ranged widely from an average of the responses to each stimulus when presented alone (Fig. 6B) (ratio $=0.5$ ) to highly superadditive (ratio $>1$ ). Across the sample, the typical bimodal effect was a summing of the responses to each stimulus alone (Fig. 6C) (median integration ratio $=1.10$ with a $95 \%$ confidence interval of [0.95,1.37], $p=0.11$, sign-test against $1, n=69)$. The scatter plot of the response to the paired stimuli against the sum of the responses to the individual stimuli (Fig. 6C) showed, similarly to previous reports (for review, see Stein and Stanford, 2008), that highly superadditive responses were observed predominantly when responses to the individual stimuli were weak, whereas response summation was observed when responses to the individual stimuli were strong. The responses to the bimodal stimuli were also plotted against the responses to $S 1_{\text {vis }}$ alone (Fig. $6 D$ ), showing that bimodal responses were generally greater than or equal to the responses to $\mathrm{S} 1_{\text {vis }}$ alone.

\section{Effect of a distant auditory competitor on visual tuning curves}

We found that auditory stimuli could also act as effective competitors to visual responses, when the auditory stimulus was located outside of the auditory RF. As with visual competitors, the effect of an auditory competitor ( $\left.\mathrm{S} 2_{\text {aud }}\right)$ on visual tuning curves was divisive. In these experiments, neuronal responses were driven with a standard $\mathrm{S} 1_{\text {vis }}$ looming stimulus presented at various azimuths across the visual RF (Fig. 7A, left panel) while an auditory stimulus (noise burst, 10-20 dB above threshold) was presented outside the auditory $\mathrm{RF}$ at a location $30^{\circ}$ lateral to the RF center.

An example is shown in Figure $7 A-D$. Responses to $S 1_{\text {vis }}$ presented alone were strong and sustained (Fig. $7 A$ ). In contrast, when the auditory competitor was presented on interleaved trials far lateral to the RF center $\left(30^{\circ}\right.$ away), responses to $S 1_{\text {vis }}$ were suppressed (Fig. $7 \mathrm{~B}, \mathrm{C}$ ). Plotting the responses to the paired auditory and visual stimuli as a function of responses to the visual stimulus alone revealed a linear relationship, demonstrating a divisive influence of the auditory competitor on responses to the $S 1_{\text {vis }}$ stimulus, and a subtractive component ( $y$-intercept) that was not significantly different from zero (Fig. 7D).

Measurements following this protocol, with the auditory competitor located $30^{\circ}$ lateral to the RF center, were made on a 
population of 14 sites (Fig. 7E). Across the population, the slope of the linear fit to the normalized data was 0.70 , indicating a substantial divisive influence. The $y$-intercept was $13 \%$ of the maximum firing rate, reflecting a slight enhancement of weak responses. There was no effect across the population on either visual RF centers or widths of visual spatial tuning ( $p>0.05$, paired $t$ tests). Thus, the primary effect of a competing auditory stimulus, like that of a competing visual stimulus, was a divisive influence on visual responses, although this particular auditory stimulus was less effective than a rapidly looming visual stimulus as a competitor (Fig. 3E).

\section{Spatial profile of auditory stimulus competition}

We also explored the dependence of stimulus competition on the location of a competing auditory stimulus (Fig. 8). For these measurements, the $S 1_{\text {vis }}$ stimulus (standard looming stimulus) was always presented near the RF center and was jittered to minimize adaptation (see Materials and Methods). On interleaved trials, the auditory competitor stimulus ( $\left.\mathrm{S} 2_{\text {aud }}\right)$ was presented simultaneously at various locations in azimuth relative to the RF center. Responses to the paired auditory and visual stimuli were plotted as a percentage change with respect to the responses to the $S 1_{\text {vis }}$ stimulus alone for the different locations of the auditory competitor.

Data from a site that was particularly strongly affected by the presence of the $\mathrm{S} 2{ }_{\text {aud }}$ stimulus are shown in Figure $8, A$ and $B$. Responses either to $\mathrm{S} 1_{\text {vis }}$ or to $\mathrm{S} 2_{\text {aud }}$ alone were strong and similarly tuned in azimuth (Fig. $8 A$ ). When the auditory and visual stimuli were presented together on interleaved trials, the auditory competitor suppressed responses to the visual stimulus from all locations outside of the auditory RF (Fig. $8 \mathrm{~B}$ ). For the most lateral location of the auditory competitor stimulus, the suppression of responses to $S 1_{\text {vis }}$ was complete (no spikes; $100 \%$ ). The suppressive effect of the auditory competitor was stronger when the competitor was located lateral to the RF center (mean suppression $=90.03 \pm$ $3.45 \%$ ) than when it was located in the opposite hemifield (mean suppression $=46.85 \pm 7.46 \% ; p=0.0002, t$ test). Auditory locations more than $\sim 15^{\circ}$ into the opposite hemifield are not represented in the space map on the same side of the brain (Knudsen, 1982).

Spatial profiles of auditory competition were measured for 14 OTi-d sites (Fig. 8C). The locations of the auditory stimulus that were consistently most effective in inhibiting responses to the visual stimulus were lateral to the RF center (Fig. $8 C$, pink); in this region, suppression averaged $43.5 \pm 6.8 \%\left(p<10^{-7}, t\right.$ test $)$. Competitor locations medial to the RF center and $>15^{\circ}$ into the opposite hemifield also suppressed responses to the $\mathrm{S} 1_{\text {vis }}$ stimulus; the suppression averaged $26.4 \pm 8.7 \%(p=0.01, t$ test $)$. When the auditory competitor was located medial to the RF cen-
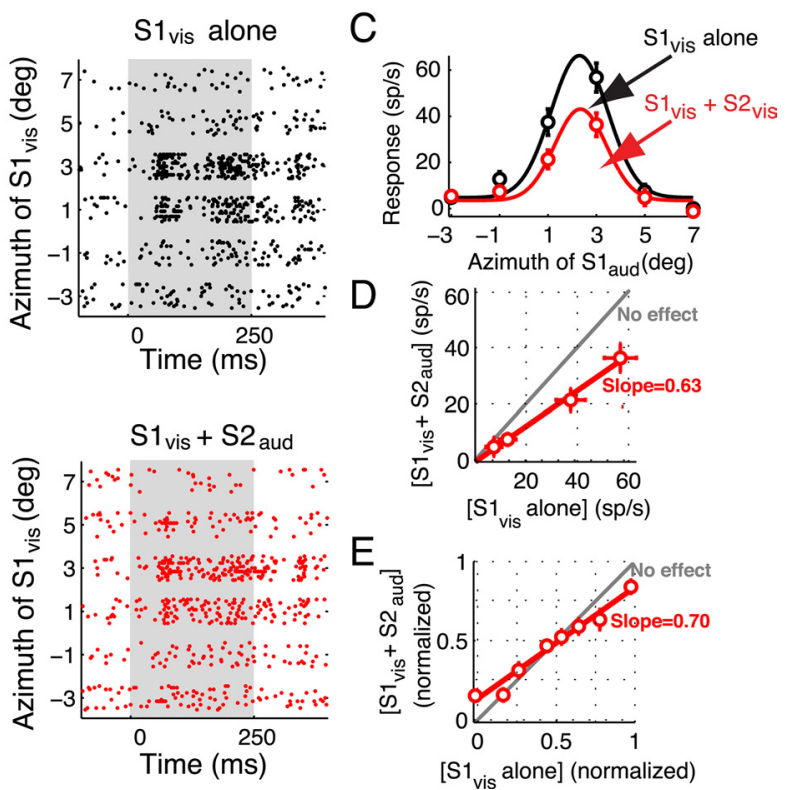

$\left[\mathrm{S} 1_{\text {vis }}\right.$ alone] (normalized)

Figure 7. Effect of a distant auditory competitor on visual tuning curves. $\boldsymbol{A}$, Left, Schematic representation of the experimenta set-up showing a single looming stimulus (black dot) at various locations. The arrows point to the responses obtained with each of 列 列 with a slope (1/divisive factor) of 0.70 (significantly different from 1, $p<0.05$; Materials and Methods), and a $y$-intercept (additive factor) of $13 \%$ in normalized coordinates (significantly different from $0, p=0.005$, see Materials and Methods).

ter, but $<15^{\circ}$ into the opposite hemifield (Fig. $8 C$, green), it did not, on average, suppress visual responses $(5.8 \pm 11 \%, p=0.62$, $t$ test), although significant suppression from this region was observed at individual sites (Fig. $8 C$, green dots). In addition, in rare instances $(5 \% ; 2 / 39$ measurements) the auditory competitor, when located medial to the RF center, facilitated responses to the $S 1_{\text {vis }}$ stimulus (Fig. $8 C$, open squares; $p<0.05$ ).

\section{Classical inhibitory surrounds}

Thus far, we have characterized the global inhibitory "surround" in the OT. In addition to global surrounds, OTi-d neurons also exhibited classical, local inhibitory surrounds. To compare and contrast the properties of these two inhibitory surrounds, we characterized classical inhibitory surrounds using both stationary and looming dot stimuli. In these experiments, neuronal responses were driven with either single stationary dots of increasing sizes or single looming dots of increasing speeds.

OTi-d neurons responded to an increase in dot size with a classic, size-response profile: an increase in responses to dots up to a certain size, followed by an asymptotic drop to a steady-state value with further increases in dot size (Fig. $1 C$, blue). This sizeresponse profile is well fit by a difference of Gaussians model (Rodieck, 1965; Sceniak et al., 1999), which accounts for the responses as a sum of an excitatory Gaussian and an aligned, but 
A
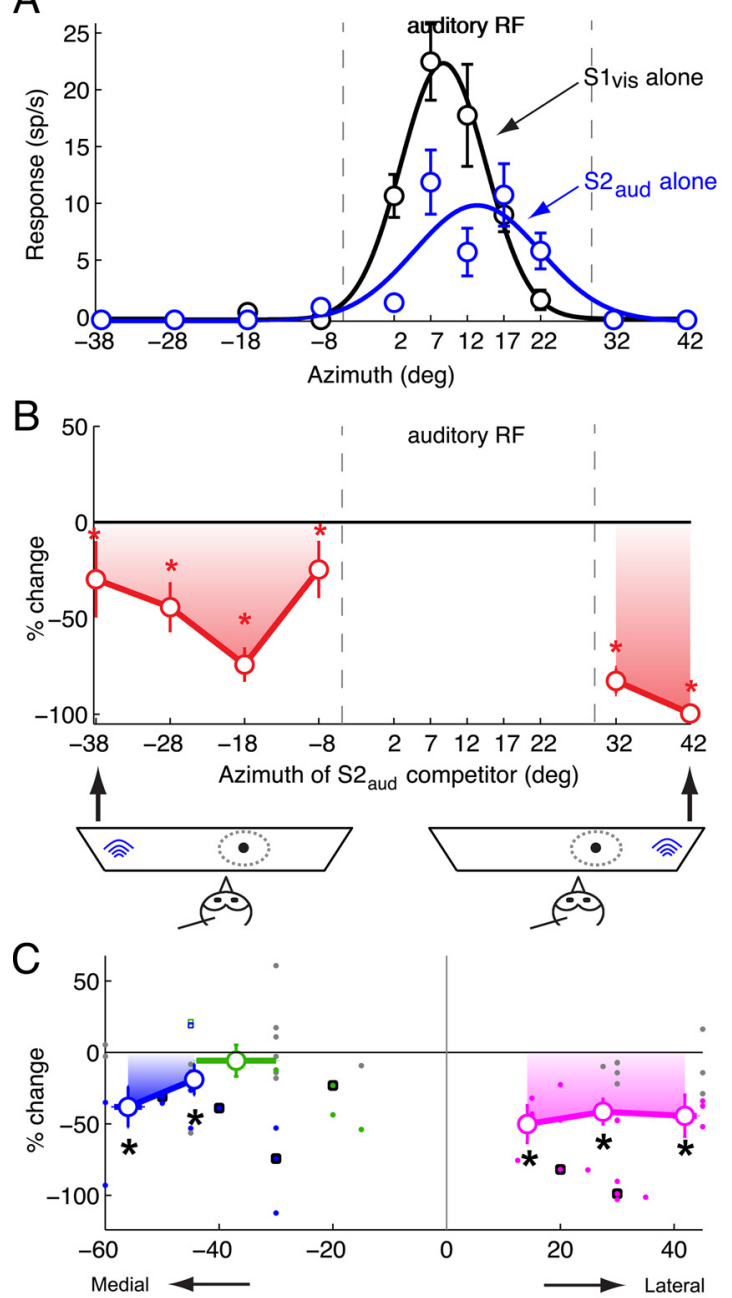

Azimuthal distance of $\mathrm{S} 2_{\text {aud }}$ competitor from RF center (deg)

Figure 8. Spatial profile of the effect of an auditory competitor on responses to a looming visual stimulus centered in the RF. A, Azimuthal tuning: responses and best-fit Gaussian profiles of an $0 \mathrm{Ti}$-d site to a single looming visual stimulus ( $\mathrm{S}_{\text {vis }}$; loom speed $=8 \%$, black) and a single noise burst auditory stimulus ( $\mathrm{S}_{\text {aud }} ; 20 \mathrm{~dB}$ above threshold noise burst; blue). Positive (negative) azimuths represent locations contralateral (ipsilateral) with respect to the recording site. The data indicate mean $\pm S E M$. $\boldsymbol{B}$, Top, Azimuth-response profile for paired $S 1_{\text {vis }}$ and $\mathrm{SZ}_{\text {aud }}$ stimuli. Site exhibited a pronounced effect in the presence of $S 2_{\text {aud }}$. Responses to $S 1_{\text {vis }}$ and $S 2_{\text {aud }}$ presented simultaneously as percentage changes with respect to the responses to the $S 1_{\text {vis }}$ stimulus presented alone. $S 1_{\text {vis }}$ was presented near the $\mathrm{RF}$ center, while $\mathrm{S} \mathrm{Z}_{\text {aud }}$ was presented at various azimuths outside the auditory RF. Asterisks indicate significant response suppression ( $p<0.05$, see Materials and Methods). Bottom, Schematic diagram showing the stimulus configuration for two extreme $S 2_{\text {aud }}$ locations. C, Summary of the effect of the azimuthal position of an auditory stimulus ( $\mathrm{S}_{\text {aud }}$ ) located outside the auditory $\mathrm{RF}$ on responses to a looming visual stimulus $\left(S 1_{\text {vis }}\right)$ presented near the $\mathrm{RF}$ center $(n=14)$. Responses are shown as percentage changes with respect to the responses to the $S 1_{\text {vis }}$ stimulus alone and are plotted as a function of the azimuthal distance of $\mathrm{SZ}_{\text {aud }}$ from the $\mathrm{RF}$ center. Only locations that were outside the auditory RF (i.e., locations at which single auditory stimuli did not drive responses; see Materials and Methods) were included. Positive values of distance represent locations lateral to the RF center; negative values represent locations medial to the RF. Filled dots indicate data from individual sites; large open circles indicate the average effect (see Materials and Methods); gray dots indicate individual data that were not significantly different from 0 (see Materials and Methods); squares with black borders indicate the data from the example site in Figure $8 B$. Locations lateral to the RF are shown in pink, those medial to the RF center and up to $15^{\circ}$ into the opposite hemifield (represented on the same side of the brain) are shown in green, locations $>15^{\circ}$ into the opposite hemifield (represented on the opposite side of the brain) are shown in blue. Asterisks indicate significant response suppression $(p<0.05$, see Materials and Methods). wider, inhibitory Gaussian (Fig. 9A, corresponding to the responses in Fig. $1 B$ ). Added together, these Gaussians yield a Mexican hat description of the classical RF: an excitatory center and an additive inhibitory surround (Fig. 9A). With this method, the radius of the classical inhibitory surround (Fig. 9A, blue curve) was estimated to be $4.9^{\circ}$ for the example site shown in Figure 1 (Fig. 9A) (see Materials and Methods). Across a population of 19 sites (same sites as in Fig. $1 D$ ), the median radius of the classical inhibitory surround measured with stationary dots was $2.6^{\circ}$, with a $95 \%$ confidence interval of $\left[2.41^{\circ}, 3.31^{\circ}\right]$, thereby verifying the limited spatial extent of the classical inhibitory surround to stationary dots.

Classical inhibitory surrounds were also measured with single looming dots using a similar procedure (Figs. 9B-D) (see Materials and Methods). Since the size of a looming dot changes over the duration of stimulus presentation, the average firing rate over that duration cannot be used to accurately estimate the size of the inhibitory surround (as it can with stationary dots). So, instantaneous firing rates were used instead. Figure $9 B$ shows the unit responses (top) and the corresponding instantaneous firing rates (bottom; see Materials and Methods) in response to a looming dot of speed of $20 \%$, from the site illustrated in Figure 1. The decline in the instantaneous firing rate after the peak (Fig. 9B) indicated the engagement of an inhibitory surround: it could not be accounted for solely by adaptation, because the unit responded with higher rates and for longer periods of time to preferred, slower looming stimuli (Fig. 9C, 16\%). The instantaneous firing rate at stimulus offset (Fig. $9 B ; r^{\text {off }}$ ) reflects the maximal spatial recruitment of the classical inhibitory surround by a looming stimulus. The spatial extent of this inhibitory surround for looming stimuli was estimated for each site to be the final dot size (radius) corresponding to the loom speed for which $r^{\text {off }}$ was within $5 \%$ of the steady-state value (Fig. $9 D$, red dashed arrow).

For the site shown in Figure 1, the estimated radius of the looming dot inhibitory surround was $9.5^{\circ}$. Across the same population of 19 sites at which stationary dots were tested (Fig. 1D), the median estimated radius of inhibitory surrounds for looming dots was $4.6^{\circ}$, with a $95 \%$ confidence interval of $\left[3.2^{\circ}, 8^{\circ}\right.$, thereby establishing the limited spatial extent of the classical inhibitory surround to looming dots. The observed difference in the sizes of the inhibitory surrounds measured with stationary and looming stimuli highlights the stimulus-dependent nature of the classical surround and is reminiscent of the stimulus-dependent changes in the dimensions of classical RFs measured in primate V1 (Kapadia et al., 1999; Sceniak et al., 1999).

\section{Constraints on the mechanisms underlying global inhibition}

The measurement of classical inhibitory surrounds revealed a novel property of the mechanisms underlying global inhibition. The responses of OTi-d neurons to stationary dots of increasing sizes (including sizes that exceeded the size of the classical inhibitory surround) asymptoted to a nonzero steady-state value [example site: Fig. $1 C, 6.8 \pm 1.6$ spikes $/ \mathrm{s}, p=0.0017, t$ test against 0 ; population data $(n=19$ sites $): 30.39 \pm 4.8$ spikes/s, $p<10^{-6}$, $t$ test against 0$]$. This indicated that increasing, symmetrical activation of the inhibitory surround resulted in a saturation of net inhibition (from both the classical surround and the global surround). The strength of inhibition due to the classical inhibitory surround diminished with distance from the RF center [Fig. 9A, inhibitory Gaussian (Somers et al., 1995; Lee and Hall, 2006]. Therefore, its contribution to net inhibition saturated with dots of large size. In contrast, the strength of inhibition due to the global surround did not diminish with distance (Fig. 5). Recon- 
ciling the nonzero asymptote of the sizeresponse profile with the unlimited extent of global inhibition requires that an additional constraint be placed on the mechanisms mediating global inhibition, one that would limit the effectiveness of a larger and larger, symmetrical stimulus in the surround. Potential candidates are (1) that neurons that mediate global inhibition mutually inhibit each other, thereby producing a saturation of global inhibitory influence when a large portion of the global network is activated (for instance, by the outer portions of a single large dot, or by an annulus), or (2) that neurons that mediate global inhibition are electrically coupled, perhaps by gap junctions (Vaney, 1999; Zhang et al., 2006).

\section{Discussion}

The results from this study demonstrate that a major determinant of neuronal responses in the OTi-d is the presence of competing stimuli in the world. Discrete, competing stimuli can be of the same or of different sensory modalities and can be located anywhere outside of the RF, including in the opposite hemifield. Competing stimuli can suppress or completely eliminate responses to an otherwise effective stimulus located inside a neuron's RF. The mechanism that mediates this competition is global, divisive inhibition that operates automatically, in a bottom-up manner, even in sedated animals. Farreaching inhibition has also been shown to operate in the cat SCi (Rizzolatti et al., 1973), but not in the cat V1 (Rizzolatti and Camarda, 1977), indicating the importance of global inhibition to information processing in the OTi-d. Although strong anesthetics can affect the balance of excitation and inhibition (Populin, 2005), the inhibitory interactions reported in this study, measured in nitrous oxide sedated owls, are consistent with those reported in the SCi of behaving monkeys (Basso and Wurtz, 1997). This argues for the validity of the results reported here.

As a result of global inhibition, activity at any location in the OTi-d represents a stimulus in the context of stimuli at all other locations. Along with additional mechanisms that may operate in behaving animals, this powerful global inhibition is likely to play a key role in the construction of a space map of stimulus salience in the optic tectum: a map that could guide attention and gaze control in behaving animals (Basso and Wurtz, 1997; Itti and Koch, 2000; Wolfe, 2003; Wolfe et al., 2003).

\section{Interactions within the $\mathrm{RF}$}

Responses to paired visual and auditory stimuli, both located inside the RF, are additive or superadditive combinations of the responses to the stimuli presented individually, consistent with the findings of previous cross-modal studies (Stein and Stanford, 2008). In contrast, responses to paired visual stimuli, both located inside the RF, are subadditive or additive combinations. This finding is consistent with previous reports of response averaging in the mammalian superior colliculus (Li and Basso, 2005;
B
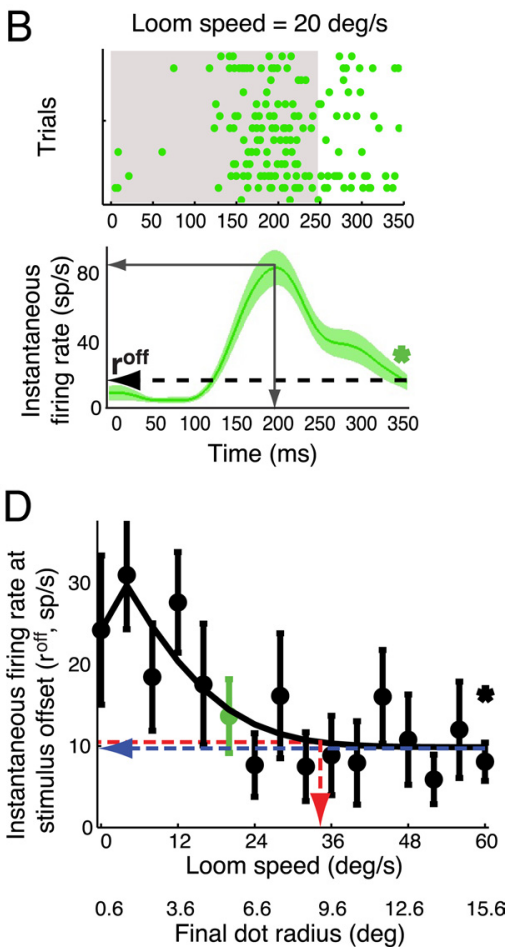

Final dot radius (deg)

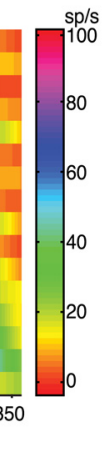

Figure 9. Classical inhibitory surrounds of 0Ti-d neurons to stationary and looming dots. $A$, Excitatory (red) and inhibitory es to a looming stimulus of speed $20 \mathrm{deg} / \mathrm{s}$ (same site as in $A$ and Fig. 1). Bottom, Mean and SEM of instantaneous fining rates from the raster (see Materials and Methods). The dashed arrow indicates firing rate at stimulus offset $\left(r^{\text {off }}\right){ }^{*} p<0.05$ ( $t$ test ainst 0). Gray, solid arrows indicate the peak firing rate and the time to peak rate. C, Pseudocolor plot of instantaneous firing rate oom speed. In green is the $r^{\text {off }}$ for a loom speed of $20 \%$, from ( $\boldsymbol{B}$, bottom panel). The steady-state value is shown in blue, and loom speed that evoked a response within $5 \%$ of the steady-state value is shown in red. The final dot radius at this loom speed is an estimate of the radius of the local inhibitory surround for a looming stimulus.

Alvarado et al., 2007) and visual cortices (Reynolds et al., 1999). Although apparently different, cross-modal and unimodal interactions in the OTi-d could still be explained by a single normalization rule-weighted averaging, with the weights that determine the contribution of each stimulus to the paired-stimulus response being $\geq 1$ in the cross-modal case and $\leq 1$ in the unimodal case. This difference in weights could reflect the fact that auditory and visual responses are processed in two independent pathways, whereas unimodal inputs are processed within a single pathway.

\section{Variations in the strength of global inhibition}

All neurons are not equally engaged by the global inhibitory network, and $\sim 10 \%$ appear not be engaged at all under our stimulus conditions. In addition, the strength of inhibition varies for a given neuron across competitor locations (Figs. 5A, 8C). This variability of inhibitory efficacy suggests an inhibitory network that consists of a population of extensively projecting elements, each of which is driven by stimuli in a limited region of space and each of which exerts its own inhibitory efficacy on OTi-d neurons.

Despite the variability of inhibitory efficacy across space, the strength of global inhibition exhibits certain spatial patterns. Physically identical stimuli, either visual or auditory, are approximately twice as effective as competitors when located laterally to the RF as compared to when they are located frontally (Fig. 5). This indicates that for owls, which are predators, stimuli along 
the horizon that are not in the line of sight have an advantage in the competition for representation in the OT space map. Second, competitors located in the contralateral hemifield exert the weakest average inhibitory effects, suggesting that inhibitory influences that originate from the opposite side of the brain tend to be less effective than those originating from the same side.

Inhibitory efficacy also appears, at first glance, to be different across competitor modalities: weaker suppression by auditory competitors than by visual ones (Figs. $7 E$ vs $3 E$, and Figs. $8 C$ vs $5 A$ ). However, this weaker suppressive influence of auditory competitors (noise burst stimuli) is most likely due to the weaker efficacy of the auditory stimuli chosen in this study: whereas the $\mathrm{S} 2_{\text {vis }}$ stimulus, when presented alone at the RF center, was, on average, $125 \%$ as effective in driving tectal units as the $\mathrm{S1}_{\text {vis }}$ stimulus, the $\mathrm{S} 2$ aud stimulus was, on average, only $48 \%$ as effective. The observation that the divisive rule for inhibition is conserved across modalities (Figs. 7E, 3E) further supports the hypothesis that the difference in the strength of inhibition between modalities is a reflection of the strength of the auditory competitor versus that of the visual competitor, rather than a modality-dependent difference.

\section{Properties of global versus classical inhibitory surrounds}

Global inhibitory surrounds and classical inhibitory surrounds differ in several fundamental respects. Inputs from the classical inhibitory surround interact additively with inputs from a unit's excitatory center (Sterling, 1975; Moors and Vendrik, 1979; Schellart et al., 1979). These interactions help to shape the tuning of neurons to single stimuli as well as their responses to multiple nearby stimuli. In contrast, inputs from the global inhibitory surround interact divisively with inputs from inside the RF. These interactions enable distant stimuli to powerfully suppress and even eliminate neuronal responses to stimuli within the RF, without changing the unit's tuning for stimuli.

In the OTi-d, classical and global inhibitory surrounds operate over very different spatial scales and exhibit different spatial profiles. We estimate that the median radius of classical inhibitory surrounds in the owl's OTi-d is $2.6^{\circ}$ to stationary stimuli and $4.6^{\circ}$ to looming stimuli. The spatial profile of this subtractive inhibition can be accounted for by a Gaussian-shaped pattern of inhibition, with inhibitory strength declining rapidly with distance from the RF center. In contrast, global inhibitory surrounds extend throughout all of space (Fig. 9B).

Yet another difference between the classical and global inhibitory networks is in their connectivity. While all the neurons that mediate global inhibition must be mutually coupled in some way (either by mutual inhibition or by electrical synapses, see Results), all the neurons that mediate the classical inhibitory surround are not. Together, the preceding observations indicate the actions of two different inhibitory networks.

\section{Different networks for global versus classical inhibitory surrounds}

Classical inhibitory surrounds are thought to be mediated by networks of local interneurons (Somers et al., 1995; Suarez et al., 1995; Lee and Hall, 2006), and the responses of OTi-d neurons to single visual stimuli, measured in this study, are consistent with the operation of such a local inhibitory network.

The global inhibitory surround in the OTi-d can potentially be mediated by networks either intrinsic to the OT or extrinsic to it. To date, however, no evidence has been found for the presence of an intrinsic source of long-range inhibition within the OT, either in the form of long-range inhibitory projections, or long- range excitatory projections to local interneurons (Lee and Hall, 2006). Three networks extrinsic to the OT that are candidates for mediating global inhibition are the retina, substantia nigra (avian homolog: TPc), and the magnocellular nucleus of the isthmic complex (Imc).

Spatially extensive, competitive interactions have been shown to occur in the retina (Lee et al., 1996; Baccus, 2007): the responses of certain types of retinal ganglion cells to stimuli inside the RF can be suppressed by small flashing spots or large-field stimuli in the extra-classical surround (Werblin and Copenhagen, 1974). Such interactions have been shown to result in contextdependent gain control and object-background discrimination. However, these retinal processes cannot account for competitive inhibitory influences demonstrated in this study that originate from far in the opposite hemifield or from auditory stimuli.

The substantia nigra has been shown to tonically inhibit the SC and to focally disinhibit it in a topographic manner, preceding a saccadic movement (Hikosaka and Wurtz, 1985b). However, the inhibition we observe here is widely projecting, rather than focal and topographic, and functions via a stimulus-driven increase rather than disinhibition. For these reasons, the substantia nigra is not a likely source of the global inhibition reported here.

The Imc, on the other hand, contains large, GABAergic neurons that receive topographic input from the OT. Imc neurons project back broadly to the OTi-d in a manner consistent with the observed global inhibition. Moreover, many Imc neurons project within the Imc as well (Wang et al., 2004), consistent with our hypothesis of mutual inhibition among the neurons of the global inhibitory network. Another distinctive structural property of Imc neurons is that, although they project back widely to the OT, they specifically do not project back to the part of the OT from which they receive input (Wang et al., 2004). This "hole" in the back projection to the OT could account for the apparent lack of a divisive influence evoked by stimuli located inside the RF.

\section{Consequences of global mutual inhibition}

The properties of global inhibition in the OTi-d are well suited to compute the relative salience of stimuli in a map of space in the OTi-d. The creation of a neural map of salience requires a means of comparing the representations of stimuli presented at multiple locations across space, in a modality and feature independent manner (Itti and Koch, 2000). Global mutual inhibition that works cross-modally, as described here, is one way for such comparisons to occur. A key, additional requirement for the generation of a map of salience is that the responses of the neurons in the map change systematically as the relative strengths of stimuli change (Itti and Koch, 2000), thereby allowing for a representation of relative strength. While the effect of the relative strength of stimuli remains to be determined, the divisive inhibition described here is a good candidate for generating a relative strengthdependent representation of stimuli across space.

\section{References}

Akaike H (1974) A new look at the statistical model identification. IEEE Trans Automat Contr 19:716-723.

Alvarado JC, Vaughan JW, Stanford TR, Stein BE (2007) Multisensory versus unisensory integration: contrasting modes in the superior colliculus. J Neurophysiol 97:3193-3205.

Baccus SA (2007) Timing and computation in inner retinal circuitry. Annu Rev Physiol 69:271-290.

Baddeley A (2003) Working memory: looking back and looking forward. Nat Rev Neurosci 4:829-839.

Basso MA, Wurtz RH (1997) Modulation of neuronal activity by target uncertainty. Nature 389:66-69. 
Carello CD, Krauzlis RJ (2004) Manipulating intent: evidence for a causal role of the superior colliculus in target selection. Neuron 43:575-583.

Cavanaugh J, Wurtz RH (2004) Subcortical modulation of attention counters change blindness. J Neurosci 24:11236-11243.

Cave KR, Wolfe JM (1990) Modeling the role of parallel processing in visual search. Cogn Psychol 22:225-271.

Dean P, Redgrave P (1984) The superior colliculus and visual neglect in rat and hamster. I. Behavioural evidence. Brain Res 320:129-141.

DeBello WM, Knudsen EI (2004) Multiple sites of adaptive plasticity in the owl's auditory localization pathway. J Neurosci 24:6853-6861.

Desimone R, Duncan J (1995) Neural mechanisms of selective visual attention. Annu Rev Neurosci 18:193-222.

Findlay JM, Walker R (1999) A model of saccade generation based on parallel processing and competitive inhibition. Behav Brain Sci 22:661-674; discussion 674-721.

Frost BJ, Scilley PL, Wong SC (1981) Moving background patterns reveal double-opponency of directionally specific pigeon tectal neurons. Exp Brain Res 43:173-185.

Hikosaka O, Wurtz RH (1985a) Modification of saccadic eye movements by GABA-related substances. I. Effect of muscimol and bicuculline in monkey superior colliculus. J Neurophysiol 53:266-291.

Hikosaka O, Wurtz RH (1985b) Modification of saccadic eye movements by GABA-related substances. II. Effects of muscimol in monkey substantia nigra pars reticulata. J Neurophysiol 53:292-308.

Itti L, Koch C (2000) A saliency-based search mechanism for overt and covert shifts of visual attention. Vision Res 40:1489-1506.

Itti L, Koch C (2001) Computational modelling of visual attention. Nat Rev Neurosci 2:194-203.

Kapadia MK, Westheimer G, Gilbert CD (1999) Dynamics of spatial summation in primary visual cortex of alert monkeys. Proc Natl Acad Sci U S A 96:12073-12078.

Kirvel RD, Greenfield RA, Meyer DR (1974) Multimodal sensory neglect in rats with radical unilateral posterior isocortical and superior collicular ablations. J Comp Physiol Psychol 87:156-162.

Knudsen EI (1982) Auditory and visual maps of space in the optic tectum of the owl. J Neurosci 2:1177-1194.

Knudsen EI (2007) Fundamental components of attention. Annu Rev Neurosci 30:57-78.

Knudsen EI, Knudsen PF, Masino T (1993) Parallel pathways mediating both sound localization and gaze control in the forebrain and midbrain of the barn owl. J Neurosci 13:2837-2852.

Koch C, Ullman S (1985) Shifts in selective visual attention: towards the underlying neural circuitry. Hum Neurobiol 4:219-227.

Lee BB, Silveira LC, Yamada E, Kremers J (1996) Parallel pathways in the retina of Old and New World primates. Rev Bras Biol 56 [Suppl $1 \mathrm{Pt}$ 2]:323-338.

Lee P, Hall WC (2006) An in vitro study of horizontal connections in the intermediate layer of the superior colliculus. J Neurosci 26:4763-4768.

Li X, Basso MA (2005) Competitive stimulus interactions within single response fields of superior colliculus neurons. J Neurosci 25:11357-11373.

Masino T, Knudsen EI (1993) Orienting head movements resulting from electrical microstimulation of the brainstem tegmentum in the barn owl. J Neurosci 13:351-370.

McPeek RM, Keller EL (2004) Deficits in saccade target selection after inactivation of superior colliculus. Nat Neurosci 7:757-763.

Meredith MA, Stein BE (1986) Spatial factors determine the activity of multisensory neurons in cat superior colliculus. Brain Res 365:350-354.

Moors J, Vendrik AJ (1979) Responses of single units in the monkey superior colliculus to stationary flashing stimuli. Exp Brain Res 35:333-347.

Müller JR, Philiastides MG, Newsome WT (2005) Microstimulation of the superior colliculus focuses attention without moving the eyes. Proc Natl Acad Sci U S A 102:524-529.

Olshausen BA, Anderson CH, Van Essen DC (1993) A neurobiological model of visual attention and invariant pattern recognition based on dynamic routing of information. J Neurosci 13:4700-4719.

Overton P, Dean P (1988) Detection of visual stimuli after lesions of the superior colliculus in the rat; deficit not confined to the far periphery. Behav Brain Res 31:1-15.
Populin LC (2005) Anesthetics change the excitation/inhibition balance that governs sensory processing in the cat superior colliculus. J Neurosci 25:5903-5914.

Raczkowski D, Casagrande VA, Diamond IT (1976) Visual neglect in the tree shrew after interruption of the descending projections of the deep superior colliculus. Exp Neurol 50:14-29.

Reynolds JH, Chelazzi L, Desimone R (1999) Competitive mechanisms subserve attention in macaque areas V2 and V4. J Neurosci 19:1736-1753.

Rizzolatti G, Camarda R (1977) Influence of the presentation of remote visual stimuli on visual responses of cat area 17 and lateral suprasylvian area. Exp Brain Res 29:107-122.

Rizzolatti G, Camarda R, Grupp LA, Pisa M (1973) Inhibition of visual responses of single units in the cat superior colliculus by the introduction of a second visual stimulus. Brain Res 61:390-394.

Rizzolatti G, Camarda R, Grupp LA, Pisa M (1974) Inhibitory effect of remote visual stimuli on visual responses of cat superior colliculus: spatial and temporal factors. J Neurophysiol 37:1262-1275.

Robinson DA (1972) Eye movements evoked by collicular stimulation in the alert monkey. Vision Res 12:1795-1808.

Rodieck RW (1965) Quantitative analysis of cat retinal ganglion cell response to visual stimuli. Vision Res 5:583-601.

Roucoux A, Guitton D, Crommelinck M (1980) Stimulation of the superior colliculus in the alert cat. II. Eye and head movements evoked when the head is unrestrained. Exp Brain Res 39:75-85.

Sceniak MP, Ringach DL, Hawken MJ, Shapley R (1999) Contrast's effect on spatial summation by macaque V1 neurons. Nat Neurosci 2:733-739.

Schellart NA, Riemslag FC, Sperkreijse H (1979) Center-surround organisation and interactions in receptive fields of goldfish tectal units. Vision Res 19:459-467.

Schiller PH, Stryker M (1972) Single-unit recording and stimulation in superior colliculus of the alert rhesus monkey. J Neurophysiol 35:915-924.

Somers DC, Nelson SB, Sur M (1995) An emergent model of orientation selectivity in cat visual cortical simple cells. J Neurosci 15:5448-5465.

Sprague JM, Meikle TH Jr (1965) The role of superior colliculus in visually guided behavior. Exp Neurol 11:115-146.

Stein BE, Stanford TR (2008) Multisensory integration: current issues from the perspective of the single neuron. Nat Rev Neurosci 9:255-266.

Sterling P (1975) Anatomical basis of receptive field properties of the superficial cells. Neurosci Res Program Bull 13:220-224.

Suarez H, Koch C, Douglas R (1995) Modeling direction selectivity of simple cells in striate visual cortex within the framework of the canonical microcircuit. J Neurosci 15:6700-6719.

Treisman A (1988) Features and objects: the fourteenth Bartlett memorial lecture. Q J Exp Psychol A 40:201-237.

Vaney DI (1999) Neuronal coupling in the central nervous system: lessons from the retina. Novartis Found Symp 219:113-125; discussion 125-133.

Viévard A, Fabre-Thorpe M, Buser P (1986) Role of the extra-geniculate pathway in visual guidance. I. Effects of lesioning the superior colliculus in the cat. Exp Brain Res 62:587-595.

Wang Y, Major DE, Karten HJ (2004) Morphology and connections of nucleus isthmi pars magnocellularis in chicks (gallus gallus). J Comp Neurol 469:275-297.

Werblin FS, Copenhagen DR (1974) Control of retinal sensitivity. 3. Lateral interactions at the inner plexiform layer. J Gen Physiol 63:88-110.

Witten IB (2008) Auditory processing in a complex spatial environment. Neurobiology. Ph.D. dissertation, Stanford University.

Wolfe JM (2003) Moving towards solutions to some enduring controversies in visual search. Trends Cogn Sci 7:70-76.

Wolfe JM, Butcher SJ, Lee C, Hyle M (2003) Changing your mind: on the contributions of top-down and bottom-up guidance in visual search for feature singletons. J Exp Psychol Hum Percept Perform 29:483-502.

Wurtz RH, Goldberg ME (1971) Superior colliculus cell responses related to eye movements in awake monkeys. Science 171:82-84.

Zhang AJ, Zhang J, Wu SM (2006) Electrical coupling, receptive fields, and relative rod/cone inputs of horizontal cells in the tiger salamander retina. J Comp Neurol 499:422-431. 